\title{
Multimodal Biometric Using Fusion of Fingerprint, Finger Knuckle Print and Palm Print
}

\author{
Shubhangi Neware \\ Assistant Professor, Shri Ramdeobaba College of Engineering \\ and Management, Nagpur (M.S.), India
}

\section{ABSTRACT}

Human recognition based on combination of various biometric traits is useful for considerably improving the performance accuracy. This paper is presenting different hand biometric traits used to identify a person. The objective of this paper is to propose multimodal biometric system using multiple biometric traits such as finger print, finger knuckle print (FKP) and palm print. The Convolution Neural Network and Softmax classifier is proposed to generate score of each modality separately and then fusion at decision level can be done. If final score is less than threshold value then person identity will be rejected. Fusion of different modalities at decision level will improve the performance of person identification system. Performance analysis is given for decision level fusion used with different modalities.

KEY WORDS: FINGER PRINT, FINGER KNUCKLE PRINT (FKP), PALM PRINT, CONVOLUTION NEURAL NETWORK (CNN), SOFTMAX.

\section{INTRODUCTION}

Use of credentials in various applications may leads to forgery. On the other hand biometric traits such as fingerprint, palm print, face and iris would not be theft, copied or lost. As a result adoption of biometrics technology is increasing rapidly in different areas. In India government has been started national identification scheme called "Aadhaar" card and it is the voluminous biometric database in the entire globe. Biometrics in reference to biological science has been studied since the early twentieth century. But now the meaning of word biometrics has been broadened to include the learning of methods for distinctly identifying or recognizing a person depending upon his/her one or more physiological or behavioral properties/traits.

\section{ARTICLE INFORMATION}

Received 14th Oct 2020 Accepted after revision 30th Dec 2020 Print ISSN: 0974-6455 Online ISSN: 2321-4007 CODEN: BBRCBA

Thomson Reuters ISI Web of Science Clarivate Analytics USA and Crossref Indexed Journal

\section{Clarivate
Analytics}

NAAS Journal Score 2020 (4.31) SJIF: 2020 (7.728)

A Society of Science and Nature Publication,

Bhopal India 2020. All rights reserved.

Online Contents Available at: http//www.bbrc.in/

Doi: http://dx.doi.org/10.21786/bbrc/13.14/15
Biometrics is the science and technology used to analyze and measure biological data of human being. Biometrics is the most accurate form of identifiers and it can greatly simplify life if used properly. Biometric systems recognize user depending on their physiological and behavioral properties/traits. Hand based biometrics is physiological biometric system. In this human hand can be used as traits to identify person. Different technologies comes under hand based biometrics is recognition of person using hand geometry, hand vein, palm, palm vein, finger print, finger geometry, knuckle print etc. Researchers are using different hand traits to identify a person. This paper is proposing an idea to use multiple modality of hand to recognize a person.

Palm print: Latent palm print identification is of growing significance in forensic applications. The palms of the person include extraordinary pattern of major lines, wrinkles and minor lines. The palm print of a person is estimated more reliable than fingerprint as it covers larger area as compared to fingerprint.

Hand vein: The blood vessels or veins unseen underside of the skin is different in persons and also steady over long period of time. A vein is responsible for flow of blood from one body part to another body part. The vascular

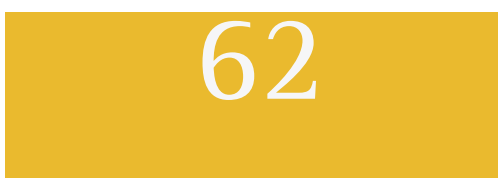


pattern is spread throughout the body which functions to supply blood in our body parts. The veins that are there in hands are simple to acquire by using infrared lighting and then that can be used for the biometric recognition or verification process.

Figure 1: Multiple Hand Traits

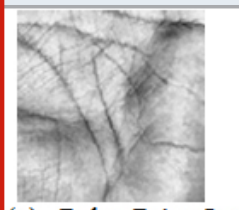

(a) Palm Print Image

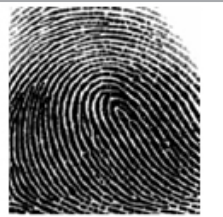

(d) Finger Print

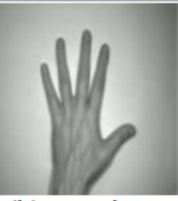

(b) Hand vein

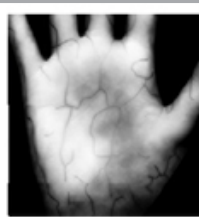

(e) Palm Vein

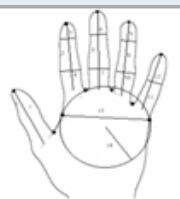

(c) Hand Geometry

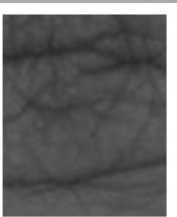

(f) Finger Knuckle
Hand Geometry: This involves the comparisons between size of fingers, position of finger joints, contour and dimension of palm. Person identification using this hand statistics use hand images to extract a number of geometrical features such as length and broadness of fingers.

Fingerprint: Fingerprint based recognition system is more accepted and successful system for person identification. It has been used for many centuries. Fingerprint consists of a texture outline of ridges positioned on tip of each finger. Landmark point on ridges is called as minutiae. It is in the appearance of ridge endings and ridge bifurcations. These minutiae point is claimed to be exceptional for each fingerprint. In fingerprint matching process comparison of 2-D minutiae patterns obtained from an individual's finger with minutiae patterns saved in the database and used for further processing.

Palm vein: Palm vein verification uses the vascular patterns formed on human palm as a biometric data for personal identification. Compared with reverse side of hand palm has broad and complicated vascular pattern which is supportive to distinguish among individuals.

Finger Knuckle Print: Texture surface pattern formed by the finger knuckle winding (knuckle joint) is called as Finger Knuckle Print (FKP). FKP can be extracted from back side of finger surface as well as inside of finger surface. FKP is extremely exclusive and make the FKP as a unique biometric modality.

Literature Review: Related to the data utilized for human recognition, earlier hand based technologies classified into palm technology, hand vein, hand geometry and hand shape technology. Many of the researchers utilized palm print for individual identification. Guagming Lu, D Zhang, K Wang has been worked on palmprint recognition using mathematical based approach. By using $\mathrm{K}-\mathrm{L}$ transform, they changed the original palmprint images into a small set of feature space. That are called "eigenpalms". The principal components i.e. significant information of the palmprints can be represented by the eigenpalms. Training set consists of eignpalms stored in the form of eigen vectors. By projecting a test palmprint image into the subspace spanned by the "eigenpalms".

Then palmprint recognition can be done using Euclidean distance classifier . Zhang D., W Kong presented an approach for online palmprint identification. A strong image coordinate system is defined by them to smooth the progress of image positioning for feature extraction. In addition to this they proposed a 2D Gabor phase encoding scheme for palmprint feature extraction and illustration. Connie et al developed Palmprint recognition using and ICA (Independent Component Analysis) and PCA (Principal Component Analysis) . By Anil K. Jain, Feng proposed similarity of Palmprint using minutiae features. Doublet, J., Lepetit 0., Revenu employed touch less hand identification by texture and shape features by combining information from color and texture.

Michael Goh Kah Ong proposed touch less palmprint biometrics using local binary pattern(LBP) texture descriptor on the palm print directional gradient responses. A Morales, M Ferrer, F Díaz, J mAlonso, C. Travieso given a touch less biometric recognition system based on human hand geometrical featutres. R Kozik, M Chora collectively taken Shape and Texture Information for Palmprint Biometrics. Finger knuckle print is identified as a new hand based biometric identifier. A Kumar and Ravikanth (2009) proposed a novel approach for human verification using finger knuckle image. Author uses texture pattern formed by the finger knuckle bending for recognition as it is extremely distinct. They employ subspace methods such as Independent Component Analysis LDA. Finger geometry features are also extracted from the same image at the same time and integrated to improve the user-identification performance. Y Zhou (2009) examines a new method for efficient individual identification using KnuckleCodes.

After preprocessing knuckle images are used to generate KnuckleCodes using Localized Radon Transform. LRT can efficiently distinguish random curved lines and creases. The similarity between two KnuckleCodes is computed from the least matching distance. A Nigam and P Gupta (2011) offered a promising FKP based identification scheme by combining Scale Invariant Feature Transform and Speeded up Robust Features. Matching Scores are then generated using fusion. The subsequent features of the enrolled Finger Knuckle Print and the test Finger Knuckle Print are matched by means of nearest neighbor ratio method and then the resultant SIFT and SURF matching scores are combined using weighted sum rule method. Vishi K, Mavroeidis V employed a fusion of fingerprint and finger-vein for person recognition by combining score of min-max, z-score, and hyperbolic tangent.

Proposed Methodology: Proposed methodology is utilizing three different modalities that are finger print, 
finger knuckle print and palm print to identify a person. In this paper Convolution Neural Network and Softmax classification technique is proposed. Three scores will be generated by three Softmax classifiers for three modalities: fingerprint, finger knuckle print and palm print. This fusion at decision level is proposed to generate final score. When final score is larger than threshold (theta) value then person will be accepted otherwise person will be rejected. All three biometric systems first

Figure 2: Work Flow Diagram for Multimodal Hand Based Biometrics

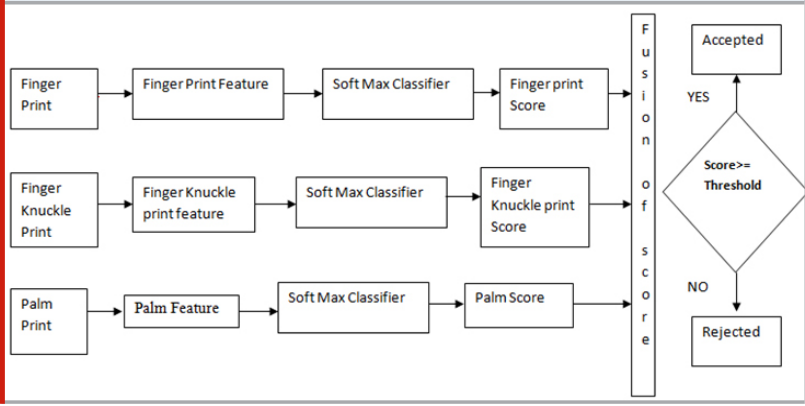

Figure 3: Working of CNN for finger print image

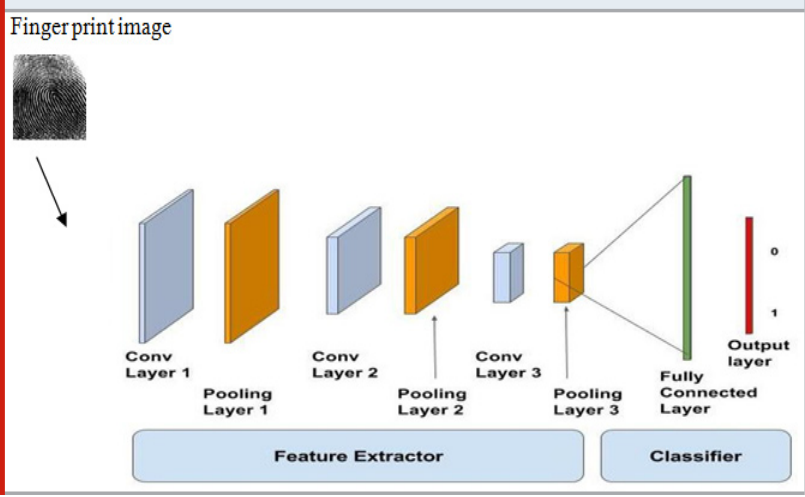

will produce their score and then decision Level fusion will take place. The decision yes or no is converted into bit representation (1-match/0-not-match). The AND-OR fusion criteria then use for outputting the last result for this biometric system working with multiple modalities. As compared to feature level fusion, combining result at decision level is less complex. Here threshold will be determined by experiment.

Convolution Neural Network: Every input image (finger print, finger knuckle and palm print) will go through a sequence of convolution layers. These layers consisting of filters called Kernals, Pooling layer and fully connected layers (FC). The function used is softmax function which categorize finger print, finger knuckle and palm print with probabilistic values in range zero to one. The figures 3 showing entire work flow of Convolution Neural Network to process an input finger print image and classifies the finger print based on values. The Softmax function which is a normalized exponential function is then used.

An input a vector $\mathrm{z}$ of $\mathrm{K}$ real numbers is input to softmax function. This function then normalized it into a probability distribution comprising of $\mathrm{K}$ probabilities. These are relatively proportional to the exponentials of the input numbers. Before giving input to softmax few vector elements could be negative or greater than one. But after applying softmax function the value will be normalized and every element in vector will within range $(0-1)$. The elements will add up to 1 , so that they can be represented as probabilities. Here the greater input elements will associate with larger probabilities. Below is an expression for defining the typical (unit) softmax function:

$$
\sigma(\mathbf{z})_{i}=\frac{e^{z_{j}}}{\sum_{j=1}^{K} e^{z_{j}}} \text { for } i=1, \ldots, K \text { and } \mathbf{z}=\left(z_{1}, \ldots, z_{K}\right) \in \mathbb{R}^{K}
$$

\begin{tabular}{|c|c|c|}
\hline Modality with Fusion & Accuracy $(\%)$ & Method ( score level fusion) \\
\hline face and finger vein & 95 & Weighted Fuzzy method \\
\hline speech and face & 99.91 & $\begin{array}{l}\text { Finite Gaussian mixture model (GMM) } \\
\text { based Expectation Maximization (EM) } \\
\text { estimated algorithm }\end{array}$ \\
\hline fingerprint and finger vein & 98.7 & Min-max normalization used for normalization. \\
\hline Iris and finger print & 94.1 & The iGRVM classifier. \\
\hline $\begin{array}{l}\text { Finger print, FKP, Palm print } \\
\text { [Proposed work] }\end{array}$ & 97 & CNN, softmax \\
\hline
\end{tabular}

\section{RESULTS}

As shown in Table I the performance analysis of various method using decision level fusion is recorded for multimodal biometric system. For combining results of two or more modalities, integration of scores is used at decision level by applying different fusion method. The accuracy of system is also improved using fusion technique of multiple modalities. Related work and their performance analysis using different type of fusion method are shown in Table1. 


\section{CONCLUSION}

In this paper various hand based traits that may be used to identify a person is introduced. Proposed work flow is given for designing of multimodal system using finger print, FKP and palm print. A multimodal system for person identification using CNN models is proposed. Decision level fusion is proposed to integrate score of these three modalities to achieve high performance. In future fusion of features can be applied to improve the performance of this multimodal system.

\section{REFERENCES}

Biometrics History, NSTC. http://www.biometrics. gov/Documents/BioHistory.pdf. Accessed 30 January 2010.

Jain A, Kumar A, (2010) Biometrics of Next Generation: An Overview", Second Generation Biometrics, Springer.

K Delac, M Grgic , "A Survey of biometric recognition methods", 46th International Symposium Electronics in Marine, ELMAR-2004, 16-18 June 2004,Zadar, Croatia.

Kumar A and Prathyusha KV (2009), "Personal authentication using hand vein triangulation and knuckle shape”, IEEE Trans Image Processing, vol. 38, no. 9: 2127-2136.

S. Neware, (Jan 2018) Biometric System: An overview”, International Journal of Computer Science and Engineering,Vol-6,issue-1,pp 313-19.

S. Neware, (2015) Finger Knuckle Identification using RLF and Dynamic Time Warping”, International Journal of Computer Applications, Vol119, issue-3.

S. Neware, K. Mehta, A.S. Zadgaonkar, (2013)Finger Knuckle Identification using Principal Component Analysis and Nearest Mean Classifier",Vol17, issue-9.

S. Neware, (2018) Finger knuckle biometrics for personal identification using statistical and feature based approaches”, International Journal of Engineering and Technology, Vol-7 (4) ,5213-5217.

S. Neware, K. Mehta, A.S. Zadgaonkar, (2014) Finger Knuckle Feature Extraction using Radonlike Features”, International Journal of Computer Science At Communication, Vol-5, pp 134-137.

T Connie, A Teoh, M Goh, D Ngo, (Nov-2003) Palmprint Recognition with PCA and ICA", Image and vision computing.

Vishi K, Mavroeidis V. 2018. An evaluation of score level fusion approaches for fingerprint and finger-vein biometrics. Available at http://arxiv.org/ abs/1805.10666. 